\title{
Schwarz Waveform Relaxation Method for the Viscous Shallow Water Equations
}

\author{
Véronique Martin \\ LAGA Université Paris XIII \\ martin@math.univ-paris13.fr
}

Summary. We are interested in solving time dependent problems using domain decomposition method. In the classical methods, one discretizes first the time dimension and then one solves a sequence of steady problems by a domain decomposition method. In this paper, we study a Schwarz Waveform Relaxation method which treats directly the time dependent problem. We propose algorithms for the viscous Shallow Water equations.

\section{Introduction}

The principle of domain decomposition methods is to partition the initial domain into several subdomains and then to use a processor per subdomain to solve the equation. The global solution is obtained if the processors exchange informations in an iterative way at the common interfaces. This method is useful to solve problems with a great number of unknowns. And it is more and more used to simulate complex phenomena with different spatial discretizations in each subdomain.

Solving time dependent problems, classical methods discretize the time dimension first and then use domain decomposition methods on the steady problems at each time step. Different strategies rely on the choice of transmission conditions (see Schwarz [1870], Lions [1990], Quarteroni and Valli [1999], Japhet et al. [2001]). In particular, in Japhet et al. [2001] transmission conditions are designed which minimize the convergence rate. This strategy proved to be very useful for many steady problems, for instance convection diffusion, Euler or Helmholtz equations. However the classical strategy to treat evolution equations does not allow to manage different time discretizations for each subdomain.

In some recent works a domain decomposition method for evolution problems quite different from the classical one has been proposed: they apply the iterative algorithm directly to the time dependent problem. This Schwarz Waveform Relaxation (SWR) method, permits to work with different time discretizations in each subdomain and therefore it provides an accurate method 
to simulate complex phenomena. This method is a derivation of the Waveform Relaxation method: inspired by the Picard iteration, it has been studied in Lelarasmee et al. [1982] for integrated circuit simulation and its convergence can be accelerated by a multigrid method (see Vandewalle [1993]).

The first SWR algorithm used Dirichlet conditions on the interfaces (see Gander and Zhao [1997], Gander and Stuart [1998]) and more recently more appropriate interface conditions have been written in Gander et al. [1999]. In this paper we apply Schwarz Waveform Relaxation methods to the Shallow Water equations.

The Shallow Water equations are obtained by average of the Navier-Stokes equations when the depth of the water is much smaller than the other dimensions of the basin. If linearized around the velocity field $\mathbf{U}=\mathbf{0}$ this model becomes (see for example Pedlosky [1987])

$$
\left\{\begin{aligned}
\partial_{t} \mathbf{U}-\nu \triangle \mathbf{U}+D \mathbf{U}+c^{2} \nabla h & =\tau_{\mathbf{s}} / \rho_{0} \\
\partial_{t} h+\operatorname{div} \mathbf{U} & =0
\end{aligned}\right.
$$

where $\mathbf{U}=(u, v)$ is the velocity field, $h$ the depth of the water, $D=\left(\begin{array}{cc}0 & -f \\ f & 0\end{array}\right)$, $c^{2}$ is the speed of internal gravity waves, $\nu$ the viscosity of the fluid, $\tau_{\mathbf{s}}$ is the wind stress and $f$ the Coriolis force supposed to be constant for the theory. We introduce the Shallow Water operator $\mathcal{L}_{S W}$ where $\mathbf{W}=(\mathbf{U}, h)$ and we are interested in solving $\mathcal{L}_{S W} \mathbf{W}=\mathbf{F}_{W}$ in $\Omega \times(0, T)$ with $T<+\infty$, $\mathbf{W}(\cdot, \cdot, 0)=\mathbf{W}_{0}$ in $\Omega$ and with boundary conditions.

In this paper we study Schwarz Waveform Relaxation algorithms to solve the Shallow Water equations. We work on the space $\mathbb{R}^{2}$ which is split into two half spaces $\Omega_{-}=(-\infty, L) \times \mathbb{R}$ and $\Omega_{+}=(0,+\infty) \times \mathbb{R}, L \geq 0$ is the overlap and let $\Gamma_{0}=\{y \in \mathbb{R}, x=0\}$ and $\Gamma_{L}=\{y \in \mathbb{R}, x=L\}$ denote the interfaces.

In Section 2 we propose an algorithm with Dirichlet interface conditions (which needs an overlap), then we propose in Section 3 an optimized algorithm which can be implemented without overlap. Finally we show numerical results which underline the efficiency of the optimized method (Sec. 4). More details about theorems will be found in Martin [2003].

\section{Classical Schwarz Waveform Relaxation Method}

Following ideas introduced in Gander and Zhao [1997] for the heat equation, we propose the following algorithm for $L>0$

$$
\left\{\begin{array} { r l } 
{ \mathcal { L } _ { S W } \mathbf { W } _ { - } ^ { k + 1 } } & { = \mathbf { F } _ { W } \text { in } \Omega _ { - } \times ( 0 , T ) , } \\
{ \mathbf { W } _ { - } ^ { k + 1 } ( \cdot , \cdot , 0 ) } & { = \mathbf { W } _ { 0 } \text { in } \Omega _ { - } , } \\
{ \mathbf { U } _ { - } ^ { k + 1 } } & { = \mathbf { U } _ { + } ^ { k } \text { on } \Gamma _ { L } \times ( 0 , T ) , }
\end{array} \left\{\begin{array}{l}
\mathcal{L}_{S W} \mathbf{W}_{+}^{k+1}=\mathbf{F}_{W} \text { in } \Omega_{+} \times(0, T), \\
\mathbf{W}_{+}^{k+1}(\cdot, \cdot, 0)=\mathbf{W}_{0} \text { in } \Omega_{+}, \\
\mathbf{U}_{+}^{k+1}
\end{array}\right.\right.
$$

where $\mathbf{F}_{W}=\left(F_{1}, F_{2}, 0\right)=(\mathbf{F}, 0), \mathbf{W}_{0}=\left(\mathbf{U}_{0}, h_{0}\right)$ and $k \geq 0$. This algorithm is initialized by $\mathbf{U}_{ \pm}^{0}$ in $\mathbf{H}^{2,1}\left(\Omega_{ \pm} \times(0, T)\right)$ such that $\mathbf{U}_{ \pm}^{0}(\cdot, \cdot, 0)=\mathbf{U}_{0}$ in $\Omega_{ \pm}$. 
We recall that we can find in Lions and Magenes [1972] the definition of anisotropic Sobolev spaces and a theorem of extension. If we use moreover a Fourier transform in $y$, a Laplace transform in $t$ and a priori estimates, then we can prove that algorithm (2) is well posed.

Theorem 1. Let $\mathbf{F}$ be in $\mathbf{L}^{2}\left(0, T ; \mathbf{L}^{2}(\Omega)\right), \mathbf{W}_{0}=\left(\mathbf{U}_{0}, h_{0}\right)$ in $\mathbf{H}^{1}(\Omega) \times H^{1}(\Omega)$. The algorithm (2) defines two unique sequences $\mathbf{W}_{ \pm}^{k}=\left(\mathbf{U}_{ \pm}^{k}, h_{ \pm}^{k}\right)$ in $\mathbf{H}^{2,1}\left(\Omega_{ \pm} \times\right.$ $(0, T)) \times H^{1,1}\left(\Omega_{ \pm} \times(0, T)\right)$ with $\nabla h_{ \pm}$in $\mathbf{H}^{1}\left(0, T ; \mathbf{L}^{2}\left(\Omega_{ \pm}\right)\right)$.

We can prove that algorithm (2) converges by computing its convergence rate written in Fourier-Laplace variables.

Theorem 2. Let $\mathbf{F}$ be in $\mathbf{L}^{2}\left(0, T ; \mathbf{L}^{2}(\Omega)\right), \mathbf{W}_{0}=\left(\mathbf{U}_{0}, h_{0}\right)$ in $\mathbf{H}^{1}(\Omega) \times H^{1}(\Omega)$. The algorithm (2) converges in $\mathbf{L}^{2}\left(0, T ; \mathbf{H}^{1}\left(\Omega_{ \pm}\right)\right) \times L^{2}\left(0, T ; L^{2}\left(\Omega_{ \pm}\right)\right)$.

It is well-known that this algorithm is not efficient: the overlap between the two subdomains is necessary and the convergence is slow. In Gander et al. [1999] interface conditions have been introduced which are more appropriate. In the next section we apply this new strategy to the Shallow Water equations.

\section{Optimized Schwarz Waveform Relaxation Method}

In this section we consider the case without overlap of the subdomains $(L=0)$ and we denote by $\Gamma$ the common interface. Since physical transmission conditions, (i.e. quantities that must be continuous through the interface) are $\mathbf{U}$ and $-\nu \partial_{x} \mathbf{U}+c^{2}(h, 0)^{t}$ we propose the algorithm

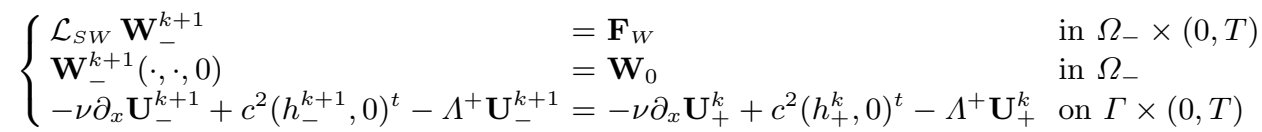

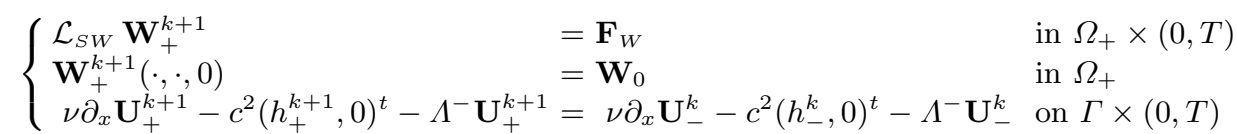

with $\Lambda^{+}$and $\Lambda^{-}$to be defined. The next theorem shows that we can choose the operators $\Lambda^{ \pm}$in an optimal way.

Theorem 3. The operators $\Lambda^{ \pm}$can be chosen such that algorithm (3) converges in two iterations. These operators are denoted $\Lambda_{\text {exac }}^{ \pm}$.

These transmission conditions coincide with absorbing boundary conditions (see for example Gander et al. [1999] for time dependent scalar equations). As for many problems the operators $\Lambda_{\text {exac }}^{ \pm}$are not differential and difficult to use, therefore we have to approximate them (see for example Nataf and Rogier [1995]). For low spatial frequencies, small Coriolis force and small viscosity $\Lambda_{\text {exac }}^{ \pm}$are approximated by: 


$$
\Lambda_{a p p}^{ \pm}=\left(\begin{array}{cc}
c+\frac{\nu}{2 c} \partial_{t} & 0 \\
0 & p
\end{array}\right),
$$

with $p$ a constant to be chosen. The following theorem gives a result of wellposedness for the corresponding algorithm. It can be proved by a FourierLaplace analysis and by an extension theorem.

Theorem 4. Let $\mathbf{F}$ be in $\mathbf{H}^{2,1}(\Omega \times(0, T)), \mathbf{W}_{0}=\left(\mathbf{U}_{0}, h_{0}\right)$ in $\mathbf{H}^{3}(\Omega) \times H^{3}(\Omega)$ and $p$ be a strictly positive constant. If algorithm (3) is initialized by $\mathbf{U}_{+}^{0}$ in $\mathbf{H}^{4,2}\left(\Omega_{ \pm} \times(0, T)\right)$ and $h_{ \pm}^{0}$ in $H^{1}\left(0, T ; H^{3}\left(\Omega_{ \pm}\right)\right)$with some compatibility relations satisfied at $t=0$, then algorithm (3) defines two unique sequences $\left(\mathbf{U}_{ \pm}^{k}, h_{ \pm}^{k}\right)$ in $\mathbf{H}^{4,2}\left(\Omega_{ \pm} \times(0, T)\right) \times H^{3,2}\left(\Omega_{ \pm} \times(0, T)\right)$ with $h_{ \pm}^{k}$ in $H^{1}\left(0, T ; H^{3}\left(\Omega_{ \pm}\right)\right)$.

By a priori estimates we can prove that algorithm (3) converges.

Theorem 5. Let $\mathbf{F}$ be in $\mathbf{H}^{2,1}(\Omega \times(0, T)), \mathbf{W}_{0}=\left(\mathbf{U}_{0}, h_{0}\right)$ in $\mathbf{H}^{3}(\Omega) \times H^{3}(\Omega)$ and $p$ be a strictly positive constant. If algorithm (3) is initialized by $\mathbf{U}_{ \pm}^{0}$ in $\mathbf{H}^{4,2}\left(\Omega_{ \pm} \times(0, T)\right)$ and $h_{ \pm}^{0}$ in $H^{1}\left(0, T ; H^{3}\left(\Omega_{ \pm}\right)\right)$with some compatibility relations satisfied at $t=0$, then the sequences $\left(\mathbf{U}_{ \pm}^{k+1}, h_{ \pm}^{k+1}\right)$ defined by (3) converge in $\mathbf{L}^{2}\left(0, T ; \mathbf{H}^{1}\left(\Omega_{ \pm}\right)\right) \times L^{2}\left(0, T ; L^{2}\left(\Omega_{ \pm}\right)\right)$.

\section{Numerical Results}

\subsection{Description of the experience}

We work on a rectangular basin with closed boundaries, which extends from 0 to $15000 \mathrm{~km}$ in the $x$ (east-west) direction and from $-1500 \mathrm{~km}$ to $1500 \mathrm{~km}$ in the $y$ (north-south) direction. The wind stress $\boldsymbol{\tau}_{s}=\left(\tau_{x}, \tau_{y}\right)$ is purely zonal $\left(\tau_{y}=0\right)$ and we have $\tau_{x}=0.5 \tau_{0}\left(1+\tanh \left(\left(x-x_{0}\right) / L\right)\right)$, with $\tau_{0}=5 \cdot 10^{-2}$ $\mathrm{N} / \mathrm{m}^{2}$ and $x_{0}=3000 \mathrm{~km}$. The value of the physical parameters are $c=3$ $\mathrm{m} / \mathrm{s}$ and $\nu=500 \mathrm{~m}^{2} / \mathrm{s}$. For further details about the experience the reader is referred to Jensen and Kopriva [1990].

The Figure 1 shows the evolution in time of the depth of water. At $t=0$ the ocean is at rest when the wind stress begins to be applied. Towards the equator the upper layer thickness increases. This anomaly travels eastward with a speed $c=3 \mathrm{~m} / \mathrm{s}$ (the speed of Kelvin waves present in the model without viscosity or external stress). After 60 days the wave reaches the eastern wall and the incoming wave is divided into four waves: two coastal Kelvin waves and two Rossby waves (see for example Pedlosky [1987] for more details about these waves).

\subsection{Solving by domain decomposition method}

We solve now this problem by domain decomposition method with the interface at $x=7500 \mathrm{~km}$. The value of the space and time steps is $\Delta x=25 \mathrm{~km}$ 

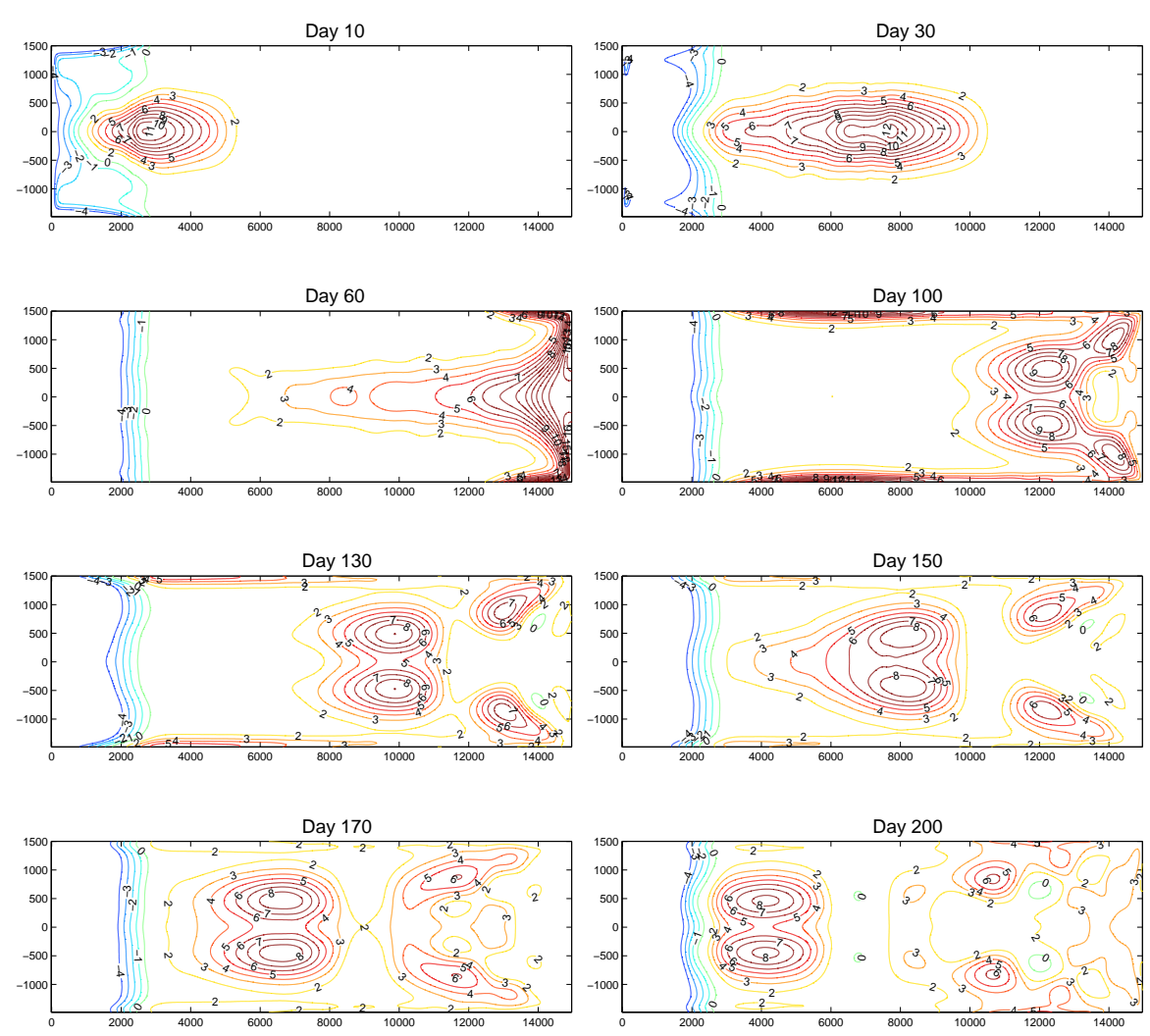

Fig. 1. Width of water at day 10, 30, 60, 100, 130, 150, 170 and 200
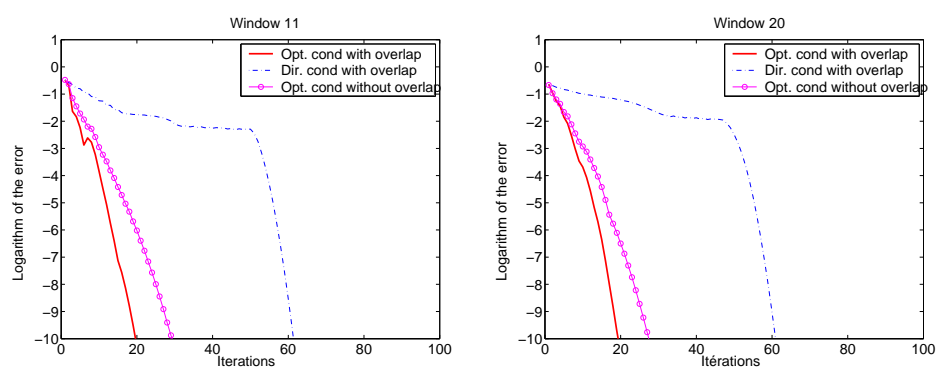

Fig. 2. Evolution of the logarithm of the error $L^{2}(\Omega)$ at the end of the time windows 11 and 20 versus the iterations

and $\Delta t=30 \mathrm{~min}$. The experience lasts 200 days, therefore $200 \times 24 \times 2=9600$ time steps are needed. Schwarz Waveform Relaxation methods work on the whole time interval, but if this one is too large, solving the equation in $(0, T)$ can be too expensive. So, we will split the time interval into several smaller 

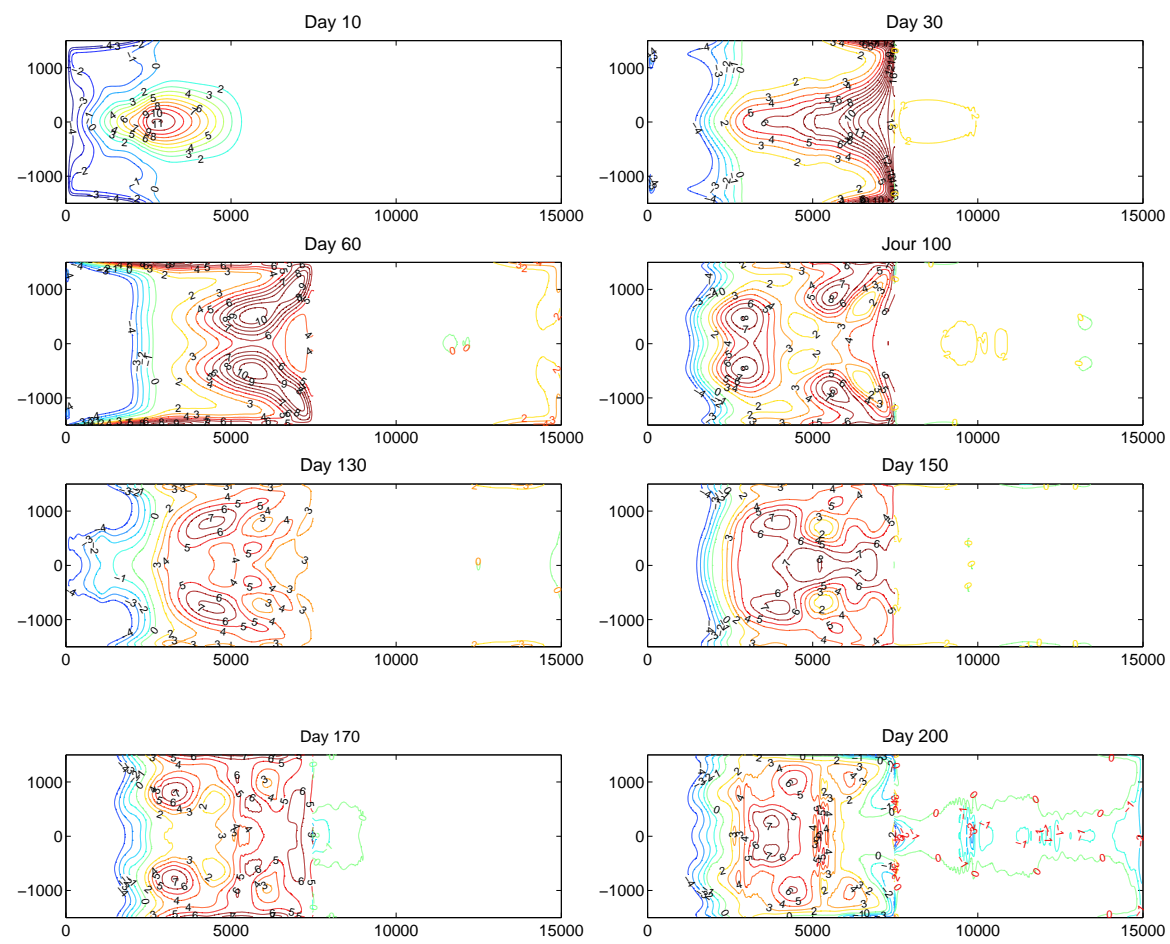

Fig. 3. Solution after two Schwarz iterations and with Dirichlet conditions at day $10,30,60,100,130,150,170$ and 200

time intervals. We write $(0, T)=\cup_{i=0, N-1}\left(T_{i}, T_{i+1}\right)$ with $T_{0}=0$ and $T_{N}=T$, then we apply our domain decomposition algorithm on each time window; we first solve $\mathcal{L}_{S W} \mathbf{W}^{0}=\mathbf{F}$ in $\Omega \times\left(0, T_{1}\right)$ with $\mathbf{W}^{0}(\cdot, \cdot, 0)=\mathbf{W}_{0}$ in $\Omega$ then for all $i \geq 1$ :

$$
\begin{cases}\mathcal{L}_{S W} \mathbf{W}^{i}=\mathbf{F} & \text { in } \Omega \times\left(T_{i}, T_{i+1}\right), \\ \mathbf{W}^{i}\left(\cdot, \cdot, T_{i}\right)=\mathbf{W}^{i-1}\left(\cdot, \cdot, T_{i}\right) & \text { in } \Omega,\end{cases}
$$

Here $T_{i+1}-T_{i}=10$ days, i.e. we are going to work with 20 windows of 10 days.

When the overlap is $L=\Delta x$, we use the Dirichlet conditions introduced in Section 2 and the optimized conditions of Section 3. When there is no overlap we can only use optimized conditions. The parameter $p$ of algorithm (3) with $\Lambda^{ \pm}=\Lambda_{a p p}^{ \pm}$optimizes the convergence rate of the algorithm (see for example Japhet [1998]). The Figure 2 shows the evolution of the logarithm of the error $L^{2}(\Omega)$ at the end of the time windows 11 and 20 versus the iterations for each method. We can see how fast is the optimized method compared to the classical Schwarz method. Obviously with an overlap the optimized method is better than without one. 

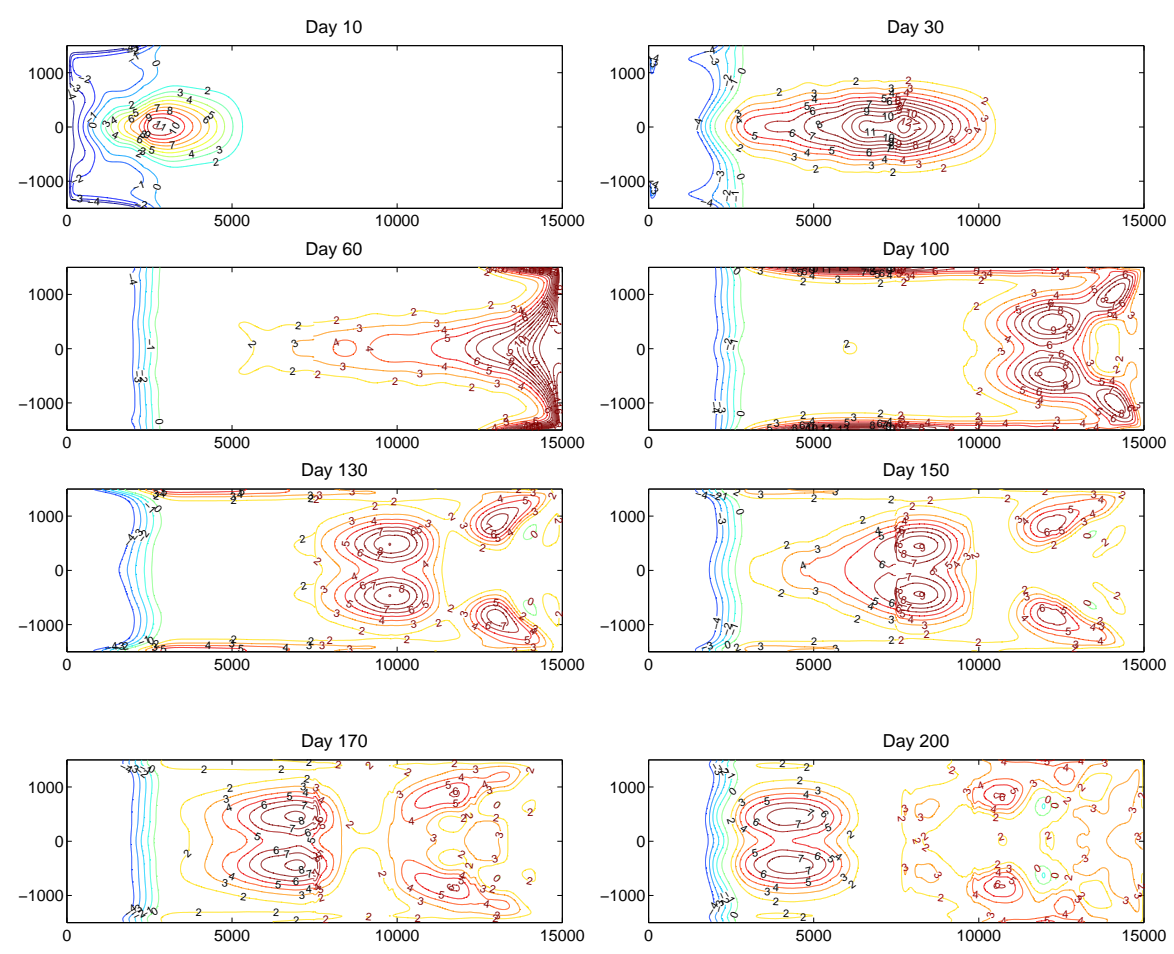

Fig. 4. Solution after two Schwarz iterations and with optimized conditions at day $10,30,60,100,130,150,170$ and 200

For more realistic simulations where such interface conditions appear, we can not wait for the convergence of the Schwarz algorithm because of the cost of each model, and only a few iterations can be implemented. The Figures 3 and 4 show the solution obtained after two Schwarz iterations in each time window with Dirichlet conditions or optimized one. We can see that Dirichlet conditions act like a wall and waves reflect in it, whereas with optimized conditions the solution is admittedly discontinuous at the interface but it is closed to the monodomain solution.

\section{Conclusion and perspectives}

We have applied a Schwarz Waveform Relaxation method to the viscous Shallow Water equations; we have studied the classical SWR algorithm and a an optimized algorithm. Numerical results have shown that the optimized method is a good one. Perspectives of that work is to improve the interface conditions of the optimized algorithm and apply this method to the Shallow Water equations linearized around any velocity field $\mathbf{U}_{0} \neq 0$. 


\section{References}

M. J. Gander, L. Halpern, and F. Nataf. Optimal convergence for overlapping and non-overlapping Schwarz waveform relaxation. In C.-H. Lai, P. Bjørstad, M. Cross, and O. Widlund, editors, Eleventh international Conference of Domain Decomposition Methods. ddm.org, 1999.

M. J. Gander and A. M. Stuart. Space time continuous analysis of waveform relaxation for the heat equation. SIAM J., 19:2014-2031, 1998.

M. J. Gander and H. Zhao. Overlapping Schwarz waveform relaxation for parabolic problems in higher dimension. In A. Handlovičová, M. Komorníkova, and K. Mikula, editors, Proceedings of Algoritmy 14, pages 4251. Slovak Technical University, September 1997.

C. Japhet. Optimized Krylov-Ventcell method. Application to convectiondiffusion problems. In P. E. Bjørstad, M. S. Espedal, and D. E. Keyes, editors, Proceedings of the 9th international conference on domain decomposition methods, pages 382-389. ddm.org, 1998.

C. Japhet, F. Nataf, and F. Rogier. The optimized order 2 method. application to convection-diffusion problems. Future Generation Computer Systems FUTURE, 18, 2001.

T. G. Jensen and D. A. Kopriva. Comparison of a finite difference and a spectral collocation reduced gravity ocean model. Modelling Marine Systems, 2:25-39, 1990 .

E. Lelarasmee, A. E. Ruehli, and A. L. Sangiovanni-Vincentelli. The waveform relaxation method for time-domain analysis of large scale integrated circuits. IEEE Trans. on CAD of IC and Syst., 1:131-145, 1982.

J.-L. Lions and E. Magenes. Nonhomogeneous Boundary Value Problems and Applications, volume I. Springer, New York, Heidelberg, Berlin, 1972.

P.-L. Lions. On the Schwarz alternating method. III: a variant for nonoverlapping subdomains. In T. F. Chan, R. Glowinski, J. Périaux, and O. Widlund, editors, Third International Symposium on Domain Decomposition Methods for Partial Differential Equations, held in Houston, Texas, March 20-22, 1989, Philadelphia, PA, 1990. SIAM.

V. Martin. Méthode de décomposition de domaine de type relaxation d'ondes pour des équations de l'océanographie. $\mathrm{PhD}$ thesis, 2003.

F. Nataf and F. Rogier. Factorization of the convection-diffusion operator and the Schwarz algorithm. $M^{3} A S, 5(1): 67-93,1995$.

J. Pedlosky. Geophysical Fluid Dynamics. Springer Verlag, New-York, 1987.

A. Quarteroni and A. Valli. Domain Decomposition Methods for Partial Differential Equations. Oxford Science Publications, 1999.

H. A. Schwarz. Über einen Grenzübergang durch alternierendes Verfahren. Vierteljahrsschrift der Naturforschenden Gesellschaft in Zürich, 15:272286, May 1870.

S. Vandewalle. Parallel multigrid waveform relaxation for parabolic problems. B. G. Teubner, Stuttgart, 1993. 